\title{
Singular Dynamics of Various Macroeconomic Sectors
}

\author{
M. KozŁowska And R. KuTneR \\ Division of Physics Education, Institute of Experimental Physics, Faculty of Physics, University of Warsaw \\ Smyczkowa Str. 5/7, PL-02-678 Warsaw, Poland

\begin{abstract}
In this work we applied our original solution of the literal Rheological Model of Fractional Dynamics of Financial Market, i.e., the time-dependent solution proportional to the Mittag-Leffler function superposed with oscillations, not only to describe the singular dynamics of financial markets but also to study the singular dynamics of various macroeconomic sectors. The approach makes possible to sufficiently estimate (among others) the time macroeconomic agents, the technical analysis of bubble and crash, which is complementary to the famous one supplying power-law superposed with log-periodic oscillations.
\end{abstract} \\ of crash as well as its order. Thus we demonstrate, perhaps useful for stock market investors as well as for various
}

PACS numbers: 89.20.-a, 89.65.-s, 89.90.+n

\section{Introduction}

This work is supplementary to our recent ones $[1,2]$ as we applied here our rheological model of fractional dynamics of financial market (RMFDFM) to well describe

- dynamics of gross domestic products of leading countries as well as

- some bubbles as well as the crash present on houses and parcels' market in United States, also bubbles and crashes

- on oil market and

- some stock markets,

i.e. to well describe the singular dynamics of quite different macroeconomic sectors.

The bubbles and crashes play a key role for capitalistic, competitive free markets [3-5] and they are their unavoidable phenomena. Therefore bubbles and crashes are the natural subject of thorough and widespreaded studies of economists, sociologists, psychologists and recently, econo- and sociophysicists.

The most fruitful seems to be the concept of the discrete scale invariance applied to stock markets and considering their crashes as a kind of criticality. As a consequence the dynamics of the market within the region preceeding a crash can be described by scale-free laws containing logarithmic periodicities [6-16] (and refs. therein). The major achievement of the approach contains, so much characteristic and useful for investors, the pecursor of a crash time.

The solution supplied by our RMFDFM is complementary to the power-law superposed with log-periodic oscillations. We applied it here to describe several empirical time series proceeding a crash, which cannot be successfully handled by the latter approach.

\subsection{Non-exponential relaxation}

The non-exponential (non-Debye) relaxation may arise from the non-Markovian type of underlying processes, i.e. from the memory effects. It was shown that fractional calculus is indeed quite natural way of incorporating memory effects $[17,18]$. Hence fractional relaxation equation, ruled by power-law kernel, presents a long-term memory. The solution that plays a dominating role in fractional relaxation problems is the Mittag-Leffler (ML) function [18]

$$
\begin{aligned}
& E_{\alpha}\left(-\left(\frac{\left|t-t_{\mathrm{c}}\right|}{\tau}\right)^{\alpha}\right) \\
& =\sum_{n=0}^{\infty} \frac{(-1)^{n}}{\Gamma(1+\alpha n)}\left(\frac{\left|t-t_{\mathrm{c}}\right|}{\tau}\right)^{\alpha n}, \quad \alpha>0,
\end{aligned}
$$

which is a straightforward generalisation of the exponential one (obtained for $\alpha=1$ ) and complementary to the Tsallis $q$-exponent [19] (here $t$ is time and $t_{c}$ is the localization of the turning point from raising to falling parts of the ML function while $\alpha$ is the shape exponent). The ML function allows interpolation [18, 20] between the corresponding stretched exponential function for the short-time limit and power-law decay for the asymptotic time (if shape exponent is smaller than 1). The former plays a crucial role in our analysis making possible to define (the analogy of) phase transitions of arbitrary orders.

\subsection{Solution supplied by rheological model of the fractional dynamics of financial market}

Modern rheology explains various aspects of the non-Debye relaxation of viscoelastic materials [20-38] mainly by using different versions of the fractional solid model 
(FSM) (named also the fractional Zener model or fractional standard linear solid). They are based on the so-called fractional elements (FEs) defined by different mechanical arrangements of constitutive elements such as springs (i.e. elastic elements) and dashpots (i.e. friction ones). These arrangements form particular ladders, trees and fractal networks (cf. corresponding figures in [21] and refs. therein), which in the limit of infinite number of constitutive elements are physical realizations of the simplest fractional constitutive differential equation (i.e. Eq. (66) in [21]) being an interpolation between Hooke's and Newton's laws.

Each FE is characterized by its own sequence of spring constants and viscosities, while given arrangement of FEs defines already the rheological (macroscopic) properties of a solid or plastic material; however, several different arrangements can define the same properties*. Different versions of fractional solid models were defined by the corresponding fractional rheological constitutive equations (FRCEs), where fractional derivatives properly replaced the ordinary ones, hence FRCEs establish corresponding, time-dependent stress-strain relations.

Our RMFDFM (being a reinterpretation of the FSM, see Table I) consider spring-dashpot pair as an analog of a single trader (investor or agent), where spring symbolizes trader's activity and dashpot his aversion to risk ${ }^{\dagger}$.

TABLE I

Correspondence between stock market quantities and the Zener model ones.

\begin{tabular}{c|c}
\hline \hline Stock market & Zener model \\
\hline stock market index & strain \\
excess demand & stress \\
volume of trade & temporal temperature \\
return per unit time & linear coefficient of expansion
\end{tabular}

In other words, the spring represents a purely emotional or irrational investor's behaviour (an undamped activity) while the dashpot defines a purely rational one (fear or aversion to risk). If spring is stretched, it means that investor ordered stocks; if it is contracted, then stocks were put up for sale, otherwise (when spring leaves unchanged) the trader is doing nothing. Let us note that the dashpot always acts against any trader's activity due to the friction or aversion to any risk. Like in the FSM, the constitutive mechanic elements (springs and dashpots) were replaced here by corresponding FEs. Hence, an arrangement of spring-dashpot pairs defines

\footnotetext{
* In the context of the stock market this means that we have to deal with multifurcation of invested capital structure which, e.g. depends on different strategies assumed by investors.

$\dagger$ This definition of trader does not exclude pathological possibilities that only the spring or only the dashpot represents some trader.
}

a network of investors, which forms a social cooperative structure for a given stock market. The model is related to field called behavioural finance since it somehow incorporates the psychological motivation of investor's behaviour. Moreover, it relates to the field called agent-based modelling of economic and social systems [39].

Saying something else, the transtion from the Zener model to its fractional counterpart means that both springs and dashpots were properly replaced by fractional elements: two of them are connected in series (called fractional Maxwell element) and it is (as a whole combined element) parallel to the third FE. Thus, we have to deal with three groups of cooperative investors, which is a reminiscence of an income distribution in society, where (roughly speaking) three essentially different prosperity groups were discovered [40-43]. Nevertheless, it is still a challenge to choose arrangements, which properly map microscopic cooperative structures of stock markets.

The literal RMFDFM deals with well-defined speculative peaks (consisting of hossa and bessa paths), where dominating behaviour of stock market results from traders' activities whose strategies are based only on a direct (on-line) observations of the market state. They are conducting a technical analysis of dynamics of stock market indices and the volume of the corresponding assets and undertake decision. They are called technical traders, chartists or noise traders. Of course, traders can mutually communicate (e.g. by using phones) exchanging informations.

To compare prediction of our model with different empirical data it is sufficient to use only the real part of the exact solution $X(t)$ of the initial-value problem for our FRCE up to the lowest order terms in the exact solution (cf. Appendix A in [1]), i.e. it is suficient to use only the following approximate expression:

$$
\begin{aligned}
& \Re X\left(\left|t-t_{\mathrm{c}}\right|\right) \approx\left(X_{0}+X_{1}\right) E_{\alpha}\left(-\left(\frac{\left|t-t_{\mathrm{c}}\right|}{\tau}\right)^{\alpha}\right) \\
& -X_{1} \cos \left(\omega\left|t-t_{\mathrm{c}}\right|\right) \cos \left(\Delta \omega\left|t-t_{\mathrm{c}}\right|\right),
\end{aligned}
$$

where terms proportional to $\omega$ as well as to $\Delta \omega$ were neglected, since for empirical data considered in this work the frequencies obey self-consistently $\omega, \Delta \omega \ll 1$ (if additionally $\Delta \omega \ll \omega$ we have to deal with a beat) and all coefficients and parameters are real; the initial value $X_{0} \stackrel{\text { def. }}{=} X(0)$ and parameter $X_{1}$ are the calibrating parameters whose values were found in the next section to be much larger than unity. By comparison predictions of expression (1.2) with empirical data we can say more about the bubble and crash dynamics (or the singular one).

\subsubsection{Long-term dynamics of gross domestic products}

Before we go to financial markets, we pay our attention to Figs. 1 and 2 were we presented gross domestic products (GDPs) of so different countries as United States and China, respectively. It is seen that both set of 
empirical data (dots) $)^{\ddagger}$ are well described by the MittagLeffler function with almost the same shape exponents (which only very little exceed 1 ) but very different relaxation times (cf. Table II). However, even this little difference (of shape exponens from a natural number) makes the crash of GDP (here of the second order) possible ${ }^{\S}$. Moreover, it is amazing that both dynamics of GDPs are practically the same differing only by the relaxation times (see Table II for comparison). Let us note that the crashes of the USA and China GDPs were predicted here for a distant future namely, for $2096 \pm 33$ and $2037 \pm 10$, respectively; both dates were obtained from very simple relation $2006+\left(t_{\mathrm{c}}-t_{\max }\right)$, where $t_{\max }=142$ [ty] or 73 [ty], respectively, are the last data points after the shrink of empirical time series.

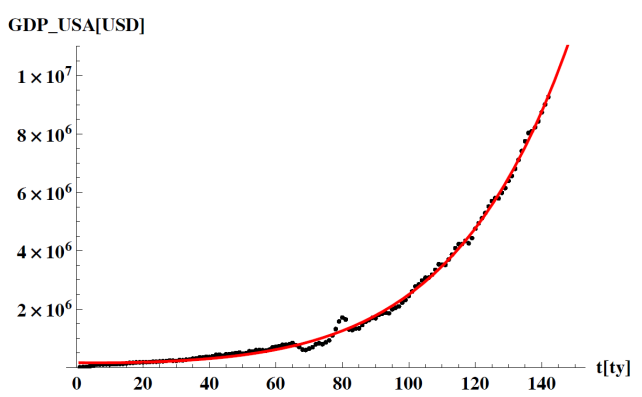

Fig. 1. The long-term U.S. gross domestic product since 1820 till 2006 normalized to 1990: (i) dots are empirical data and (ii) the solid curve is the fit given by the Mittag-Leffler function (1.1), where shape exponent $\alpha$ only slightly exceeds 1 (see Table II). Time $t$ is measured here in trading years (ty) and empirical data were shrunk discarding the missing trading years (as it mainly concerns the beginning of the empirical data set, it has a minor influence on the fit). The maximal value of empirical data is placed at $t=t_{\max }=142$

\subsubsection{Dynamics of houses and parcels' market}

Next, we pay our attention to very interesting example of the dynamics of houses and parcels' market in United States between January 1963 and December 2008; the corresponding empirical data (in $\log -\log$ scale) were shown in Fig. 3. It is clear that the whole data's range is naturally divided into two regions characterized by two essentially different power-laws:

(i) the first region (having slope equal to $0.076 \pm 0.015$ ), since January'63 untill 1970 " when Federal Home Loan Mortgage Corporation (HLMC, known as

$\ddagger$ The empirical data were downloaded from the internet address http://www.ggdc.net/Maddison/Historical_Statistics .

$\S$ Note that only $\alpha$ exactly equal to a natural number gives no crash, but this is the marginal case.

I This date is placed in Fig. 3 near the turning point where empirical data changes its slope in $\log -\log$ scale from low to high value.

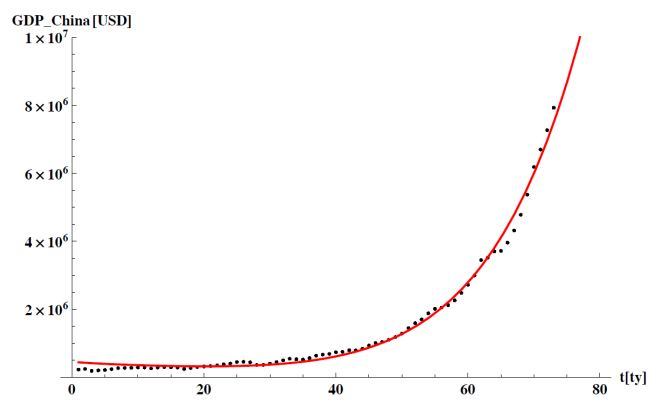

Fig. 2. The long-term gross domestic product of China since 1820 till 2006 normalized to 1990: (i) dots are as usual the empirical data and (ii) the solid curve is the fit given by the Mittag-Leffler function (1.1), where again shape exponent $\alpha$ slightly exceeds 1 (see Table II). Time $t$ is also measured here in trading years (ty) and empirical data were shrunk discarding the missing trading years (as it mainly concerns the beginning of the empirical data set, it has a minor influence on the fit). The maximal value of empirical data is placed at $t=t_{\max }=73$

TABLE II

Values of parameters found by the fit.

\begin{tabular}{c|c|c|c|c}
\hline \hline Country & $\alpha$ & $\tau$ [ty] & $t_{\mathrm{c}}[\mathrm{ty}]$ & $R^{2}$ \\
\hline USA & $1.04 \pm 0.03$ & $40 \pm 5$ & $232 \pm 33$ & 0.9987 \\
China & $1.05 \pm 0.03$ & $16 \pm 1$ & $104 \pm 10$ & 0.9956
\end{tabular}

Freddie Mac) was created to expand the secondary market for mortgages, and

(ii) the second one after the 1970 (having slope equal to $1.391 \pm 0.015)$.

Note the Freddie Mac is the United States federal government-sponsored enterprise which deals with mortgage lenders to support them get optimal access to house financing. Since 1970, Freddie Mac has systematically extended its activity beginning from rich society class over middle to poor ones creating a kind of mortgage lenders' hierarchy.

Besides Freddie Mac, there is also complementary enterprise Federal National Mortgage Association (FNMA, commonly known as Fannie Mae) sponsored by government since 1968. Its corporation's purpose is to purchase and securitize mortgages in order to ensure that funds are consistently available to the institutions that lend money to house buyers. Both enterprises play together the decisive role on a mortgage market.

In fact, we commence our analysis of empirical data by dividing them into three independent ranges according to, well-seen in the scale of Fig. 3, the fine structure of price dynamics and by beginning the first range since April 1973 (cf. Fig. 6). As we will see, although full data range can be fitted by a single power-law, each range separately is well-fitted by the Mittag-Leffler function (cf. solid curves in Figs. 4-6). 


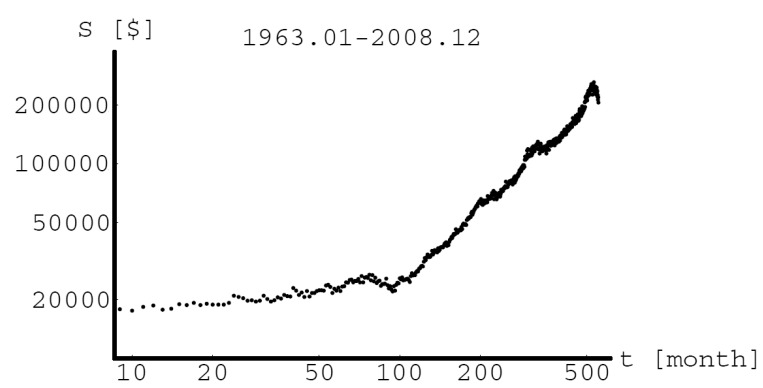

Fig. 3. The median, $S$, of houses and parcels's prices sold in U.S. between January 1963 and December 2008 shown in $\log -\log$ scale (the unit of timescale is chosen here as one trading month). The empirical data (marked by dots) were downloaded from the internet address http://www.economagic.com/ .

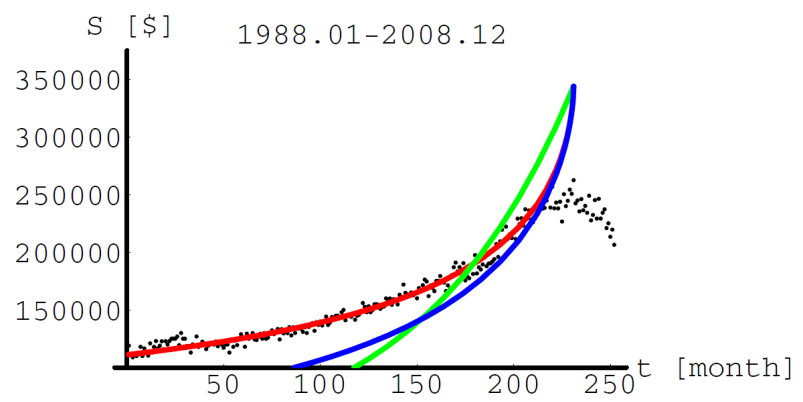

Fig. 4. The median, $S$, of prices of houses and parcels sold in US between January 1988 and December 2008 (the unit of timescale is chosen here as one trading month). The empirical data (marked by dots) were downloaded from the internet address http://www.economagic.com/ (solid curves were defined in the main text).

As it is seen, in Fig. 4 the empirical data proceeded from the market of houses and parcels is well fitted by the Mittag-Leffler function (1.1) (the best fitted curve) even near the turning point (i.e. the point where hossa changes to bessa). The values of parameters of ML function obtained here are as follows: the localization of the theoretical turning point (the maximum) is at $t_{\mathrm{c}}=231$ months, i.e. at March'07, which is placed exactly at the maximum of empirical data, the relaxation time $\tau=95$ months, the shape parameter $\alpha=0.60^{* *}$.

Let us note that for $\alpha<1$ both derivatives (i.e. leftand right-sided) of the ML function diverges at $t_{\mathrm{c}}$ according to the power-law, which means that return also accordingly diverges [2]. Hence, at $t_{\mathrm{c}}$ we deal with analogy of the first order phase transition. The corresponding predictions of exponential function (the highest lying curve in the vicinity of the top) and stretched exponen-

\footnotetext{
** All values of fittted parameters shown in this paragraph are bur-
} dened by the order of $10 \%$ errors.

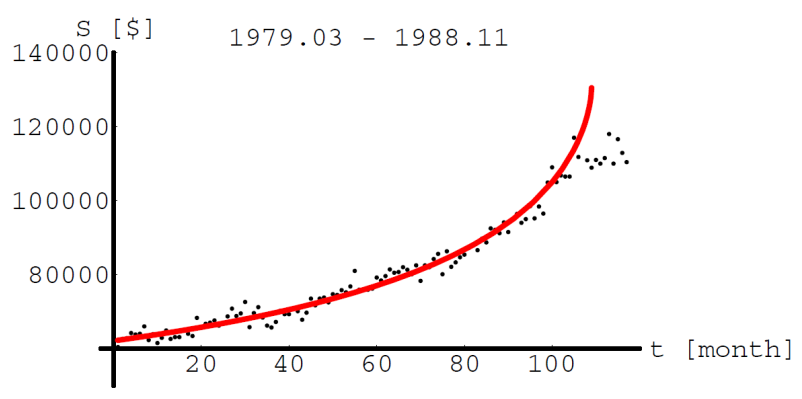

Fig. 5. The median, $S$, of prices of sold houses and parcels in US from March 1979 untill November 1988. The solid curve shows the prediction of the ML function for $\alpha=0.62, \tau=76$ months and its maximum $t_{\mathrm{c}}$ is placed at March 1988.

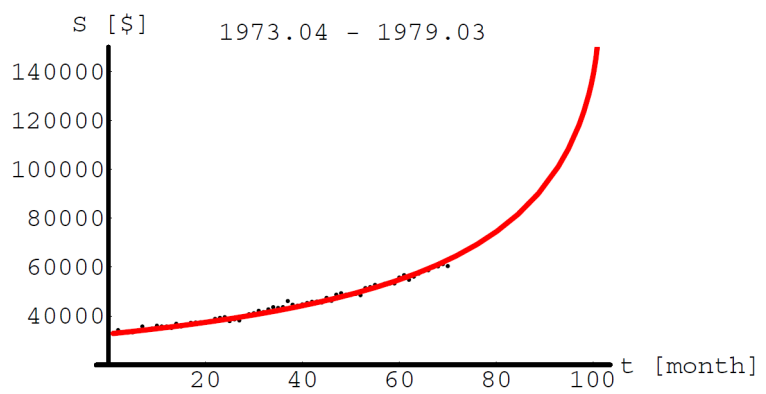

Fig. 6. The median, $S$, of prices of sold houses and parcels in USA from April 1973 untill March 1979. The solid curve shows the prediction of the ML function for $\alpha=0.65, \tau=30$ months and its maximum $t_{\mathrm{c}}$ is placed at February 1979.

tial one (the lowest lying curve) were also shown there for comparison ${ }^{\dagger \dagger}$.

In Figs. 5 and 6 good agreement between predictions of ML function and empirical data for both earlier time-ranges were also obtained.

The observation that the Mittag-Leffler function so well fits empirical data means that the (analogy of) phase transition or crash is potentially included in the market of houses and parcels in US. Whether crash really will appear depends on how far the market reaches the scaling (crash) region.

Quite often the ML function appears both in the stochastic and deterministic modelling of disordered systems. The canonical example of the former may be the continuous-time random walk model (CTRW) used in the context of a financial market [31-46], while the latter already mentioned above fractional relaxation equation, describes relaxation of viscoelastic materials.

$\dagger^{\dagger}$ For both exponential and stretched exponential functions relaxation time is given here by $\tau[\Gamma(1+\alpha)]^{1 / \alpha}=79$ months. 
1.2.3. Mittag-Leffler function superposed with oscillations on the oil market

Another, very prominent example of application of the RMFDFM's solution (1.2) is the crash on the oil market in June 2008 (cf. Fig. 7); the theoretical crash point $t_{\mathrm{c}}$

TABLE III

Fit parameters describing the recent, largest peak of the oil price. The fit accuracy $R^{2}=0.9931$.

\begin{tabular}{c|c|c|c|c}
\hline \hline$t_{\mathrm{c}}$ & $\tau$ & $\alpha$ & $\omega$ & $\Delta \omega$ \\
\hline 124.1 & 17.2 & 0.89 & 0.0215 & 0.0738 \\
\pm 0.6 & \pm 0.5 & \pm 0.01 & \pm 0.0004 & \pm 0.0010
\end{tabular}

\section{TABLE IV}

Calibrating fit parameters concerning the oil market crash on June 2008.

\begin{tabular}{c|c}
\hline \hline$X_{0}+X_{1}[p]$ & $X_{1}[p]$ \\
\hline $153.4 \pm 6.2$ & $-25.5 \pm 2.1$
\end{tabular}

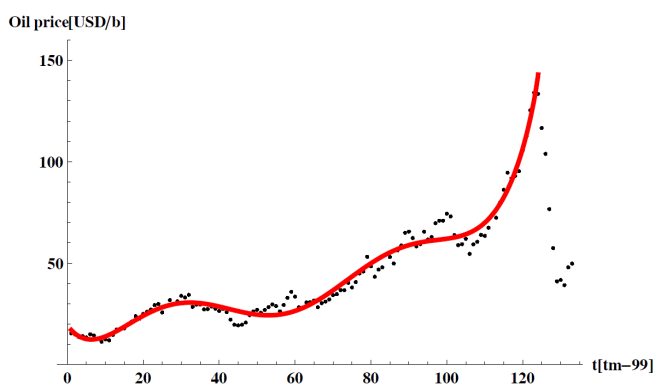

Fig. 7. Oil price peak in "USD per barrel" (marked by dots) extended from April 1998 till April 2009 (which cover 133 empirical data points; the unit of timescale is chosen here as one trading month) while the crash point (i.e. the maximum of empirical peak) is placed at June 2008 (i.e. at the 123rd day of the hossa). The empirical data were downloaded from the internet address http://www.economagic.com/ (theoretical, solid curve was defined in the main text by Eq. (1.2)).

was found by the fit on July 2008. Optimal parameters obtained from the fit were shown in Tables III and IV. As it is seen,

(i) the crash is here of the first order as shape exponent $\alpha<1$,

(ii) dispersions of all parameters is surprisingly small (of the order of few percents), for example, the theoretical crash point $t_{\mathrm{c}}$ appeared (in practice) at the same time as the empirical one.

We found that:

(iii) our aproach has ability (by direct extension) to the short-term, let us say a single quarter, forecast.

\section{Comparison with stock market empirical data and conclusions}

For literal verification of validity of our RMFDFM, one can fit the formula (1.2) to empirical data forming recent peak of index WIG20 (cf. Fig. 8 and Table V). It is seen that the fit (depicted by the solid curve and considered as a trend) is satisfactory and the distribution of empirical points around the trend seems to have the statistical character.

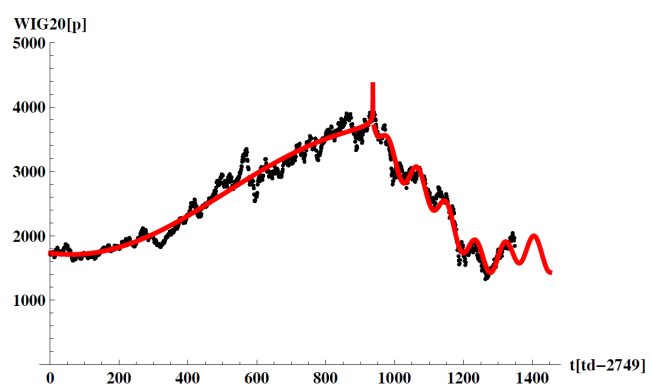

Fig. 8. A recent full peak of WIG20 extended from 2004-02-09 to 2009-06-22 (or from 2440th stock market session to the 3787 th one). It is seen, how sudden and sharp is the theoretical prediction of the peak.

However, the relaxation time $\tau$ concerning the hossa (cf. Table V) is burdened with huge dispersion. This case (observed within our deterministic approach) seems to be a typical one for any hossa (see relaxation times shown in Table $\mathrm{V}$ for S\&P500 and NASDAQ). It suggests the existence of a financial uncertainty principle (FUP) of quantities substantial to reach a profit by any investor ${ }^{\ddagger \ddagger}$. This principle can be formulated as follows: among quantities which values have to be known to reach a profit during the hossa, at least one is unmeasurable, i.e. its value cannot be determined with sufficient precision. Otherwise, the profit could be obtained without any risk which would be in contradiction to the market paradigm saying that market eliminates the arbitrage opportunity. Fortunately, due to anticorrelations existing between these quantities, the summarized dispersion can be sufficiently small to make the total fit satisfactory.

Moreover, we found that in this case the shape exponent $\alpha$ is much smaller than 1 (having large dispersion, cf. the corresponding number shown in Table V). Hence, the fitted (solid) curve violently accelerates when it moves toward $t_{c}$, where its derivative diverges. It suggests that we have to deal at this singular point with the analogy of the first order phase transition on the Warsaw Stock Exchange. Below, we extended this discussion by considering recent daily peaks of S\&P500 and NASDAQ.

$\ddagger \ddagger$ Uncertainty, together with risk and profit, were thoroughly and widespreadly reviewed in book [39]. 
Full peaks of these indices were presented in subsequent Figs. 9 and 10, while the corresponding fit parameters were shown in Tables V-VII. Again, satisfactory agreement with empirical data are observed, which makes possible to draw some common conclusions.

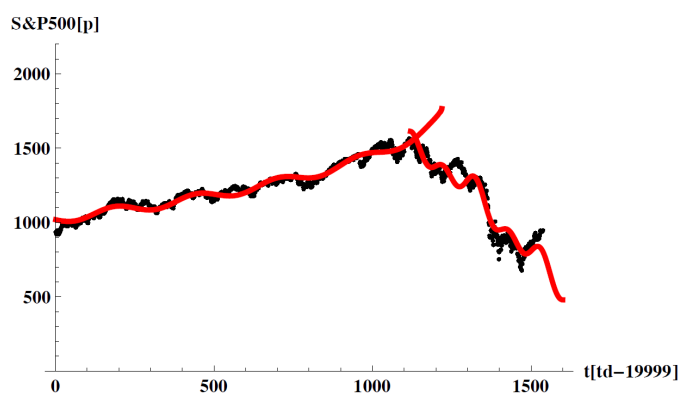

Fig. 9. A recent full peak of S\&P500 extended from 2003-05-07 to 2009-06-12 (or from 20000 stock market session to 21536 one).

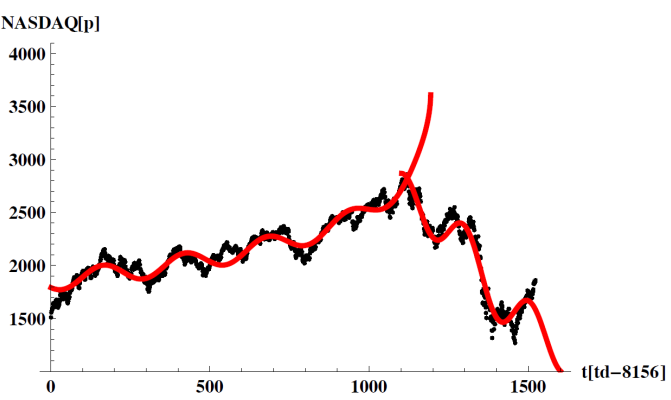

Fig. 10. A recent full peak of NASDAQ extended from 2003-05-23 to 2009-09-06 which covers 1521 stock market sessions.

TABLE V

Fit parameters describing recent peaks of typical main indices of small, middle and large stock markets (upper elements $L$ and $R$ labeling parameters, designate left and right paths of a peak, respectively).

\begin{tabular}{c|c|c|c}
\hline \hline Parameter & WIG20 & S\&P500 & NASDAQ \\
\hline$t_{\mathrm{c}}^{\mathrm{L}}$ & $937 \pm 1$ & $1219 \pm 4$ & $1193 \pm 43$ \\
$t_{\mathrm{c}}^{\mathrm{R}}$ & $940 \pm 0$ & $1120 \pm 0$ & $1105 \pm 0$ \\
$\tau^{\mathrm{L}}$ & $9967 \pm 155981$ & $1482 \pm 1681$ & $298 \pm 541$ \\
$\tau^{\mathrm{R}}$ & $218 \pm 31$ & $323 \pm 51$ & $282 \pm 39411$ \\
$\alpha^{\mathrm{L}}$ & $0.134 \pm 0.138$ & $0.72 \pm 0.19$ & $0.595 \pm 0.121$ \\
$\alpha^{\mathrm{R}}$ & $0.861 \pm 0.018$ & $1.60 \pm 0.47$ & $2.29 \pm 6.32$ \\
$\omega^{\mathrm{L}}$ & $0.00361 \pm 0.00003$ & $0.0114 \pm 0.0007$ & $0.0131 \pm 0.0003$ \\
$\omega^{\mathrm{R}}$ & $0.0472 \pm 0.0001$ & $0.0146 \pm 0.0003$ & $0.020 \pm 0.010$ \\
$\Delta \omega^{\mathrm{L}}$ & $0.0269 \pm 0.0001$ & $0.0129 \pm 0.0007$ & $0.0113 \pm 0.0003$ \\
$\Delta \omega^{\mathrm{R}}$ & $0.007 \pm 0.0$ & $0.0461 \pm 0.0003$ & $0.012 \pm 0.010$
\end{tabular}

It is seen from Table $\mathrm{V}$ that

- the range of the hossa shape exponent $\alpha$ is larger than 0 and smaller than 1 for considered peaks,
TABLE VI

Calibrating fit parameters describing peaks of the same indices as shown in Table V.

\begin{tabular}{c|c|c|c}
\hline \hline Parameter [p] & WIG20 & S\&P500 & NASDAQ \\
\hline$X_{0}^{\mathrm{L}}+X_{1}^{\mathrm{L}}$ & $1957 \pm 977$ & $1204 \pm 59$ & $1931 \pm 458$ \\
$X_{0}^{\mathrm{R}}+X_{1}^{\mathrm{R}}$ & $3145 \pm 49$ & $4010 \pm 110$ & $1193 \pm 43$ \\
$X_{1}^{\mathrm{L}}$ & $-890 \pm 21$ & $61 \pm 3$ & $-181 \pm 7$ \\
$X_{1}^{\mathrm{R}}$ & $-421 \pm 19$ & $-866 \pm 81$ & $-440 \pm 32$
\end{tabular}

TABLE VII

Accuracy of the fit, where fit parameters were shown in Tables V and VI

\begin{tabular}{c|c|c|c}
\hline \hline Fit accuracy & WIG20 & S\&P500 & NASDAQ \\
\hline$R_{\mathrm{L}}^{2}$ & 0.9978 & 0.9994 & 0.9987 \\
$R_{\mathrm{R}}^{2}$ & 0.9970 & 0.9996 & 0.9978
\end{tabular}

- localization of maxima, $t_{\mathrm{c}}$ 's, of hossa and bessa were found with relatively small dispersion in contradiction to fractional relaxation times, $\tau$ 's. For the WIG20 $t_{\mathrm{c}}^{\mathrm{R}}<t_{\mathrm{c}}^{\mathrm{L}}$ while for two remaining indices $t_{\mathrm{c}}^{\mathrm{R}} \geq t_{\mathrm{c}}^{\mathrm{L}}$.

- all frequencies, $\omega$, and frequency shifts, $\Delta \omega$, are much smaller than 1 , which is a constraint required for application of the approximate solution (1.1).

It is evident from Table VI that

- the calibration parameter $X_{1}$ can assume both the positive and negative values.

From Table VII it follows that:

- accuracies, $R^{2}$, of all fits are satisfactory as they are restricted to rather narrow range $0.9970 \leq R^{2} \leq$ 0.9996 .

We can conclude that:

- the rough short-term forecasting made by simple extension of theoretical curves is, of course, possible (see, for instance, Fig. 8).

We hope that results presented here and in our recent works $[1,2]$ pointed out at least a technical utility of the RMFDFM to study a singular evolution of various sectors of economic systems.

TABLE VIII

Dates which correspond to $t_{\mathrm{c}}^{\mathrm{L}}$ and $t_{\mathrm{c}}^{\mathrm{R}}$ shown in Table $\mathrm{V}$.

\begin{tabular}{c|c|c|c}
\hline \hline Date & WIG20 & S\&P500 & NASDAQ \\
\hline$t_{\mathrm{c}}^{\mathrm{L}}$ & $2007-10-25$ & $2008-03-11$ & $2008-02-19$ \\
$t_{\mathrm{c}}^{\mathrm{R}}$ & $2007-10-29$ & $2007-07-12$ & $2007-10-11$
\end{tabular}




\section{Acknowledgments}

Authors are indebted to Didier Sornette for stimulating discussions.

\section{References}

[1] M. Kozłowska, A. Kasprzak, R. Kutner, Int. J. Mod. Phys. C 19, 453 (2008).

[2] M. Kozłowska, R. Kutner, Acta Phys. Pol. B 37, 3027 (2006).

[3] D. Sornette, Why Stock Markets Crash. Critical Events in Complex Financial Systems, PUP, Princeton 2003.

[4] B.M. Roehner, Patterns of Speculation. A Study in Observational Econophysics, CUP, Cambridge UK 2002.

[5] M. Ausloos, in: Econophysics and Sociophysics. Trends and Perspectives, Eds. B.K. Chakrabarti, A. Chakraborti, A. Chatterjee, Wiley-VCH Verlag, Weinheim 2006, Ch. 9, p. 249.

[6] W.-X. Zhou, D. Sornette, Physica A 330, 543 (2003).

[7] W.-X. Zhou, D. Sornette, Physica A 330, 584 (2003).

[8] P. Gnaciński, D. Makowiec, Physica A 344, 322 (2004).

[9] S. Drożdż, F. Grummer, F. Ruf, J. Speth, Physica A 324, 174 (2003).

[10] S. Drożdż, J. Kwapień, P. Oświęcimka, J. Speth, Acta Phys. Pol. A 114, 539 (2008)

[11] D. Sornette, A. Johansen, J.-P. Bouchaud, J. Phys. I (France) 6, 167 (1996).

[12] D. Sornette, A. Johansen, Physica A 245, 411 (1997).

[13] D. Sornette, A. Johansen, Quant. Finance 1, 452 (2001).

[14] A. Johansen, D. Sornette, J. Risk 4, 69 (2001).

[15] A. Johansen, Europhysics Lett. 60, 809 (2002).

[16] D. Grech, Z. Mazur, Acta Phys. Pol. B 36, 2403 (2005).

[17] P.L. Butzer, U. Westphal, An Introduction to Fractional Calculus in: Fractional Calculus in Physics, Ed. R. Hilfer, World Sci., Singapore 2000.

[18] R. Metzler, J. Klafter, Phys. Rep. 339, 1 (2000).

[19] C. Tsallis, Braz. J. Phys. 39, 337 (2009).

[20] Th.F. Nonnenmacher, R. Metzler, Applications of Fractional Calculus Techniques to Problems in Biophysics, in: Applications of Fractional Calculus in Physics, Ed. R. Hilfer, World Sci., Singapore 2000.

[21] H. Schiessel, Chr. Friedrich, A. Blumen, Applications to Problems in Plymer Physics and Rheology in Fractional Calculus in Physics, Ed. R. Hilfer, World Sci., Singapore 2000, Ch. VIII.

[22] H. Schiessel, A. Blumen, J. Phys. A, Math. Gen. 26, 5057 (1995)

[23] W.G. Glöckle, Th.F. Nonnenmacher, Macromolecules 24, 6426 (1991).

[24] W.G. Glöckle, T.F. Nonnenmacher, Rheol. Acta 33 337 (1994).
[25] R. Badii, A. Politi, Complexity. Hierarchical structures and scaling in physics, Cambridge Univ. Press, Cambridge UK 1997.

[26] W. Paul, J. Baschnagel, Stochastic Processes. From Physics to Finance, Springer-Verlag, Berlin 1999.

[27] R.N. Mantegna, H.E. Stanley, An Introduction to Econophysics: Correlations and Complexity in Finance, CUP, Cambridge UK 2000.

[28] J.-P. Bouchaud, M. Potters, Theory of Financial Risks. From Statistical Physics to Risk Management, CUP, Cambridge UK 2001.

[29] K. Ilinski, Physics of Finance. Gauge Modelling in Non-equilibrium Pricing, Wiley, Chichester 2001.

[30] E. Scales, R. Gorenflo, F. Mainardi, Phys. Rev. E 69, 011107-1 (2004).

[31] L. Sabatelli, S. Keating, J. Dudley, P. Richmond, Eur. Phys. J. B 27, 273 (2002).

[32] Nonextensive statistical mechanics: new trends, new perspectives, Europhysicsnews 36/6 (2005), Special Issue \& Directory.

[33] F. Schweitzer, Brownian Agents and Active Particles, Springer-Verlag, Berlin 2003.

[34] A. Bunde, J.W. Kantelhardt, Physikaliche Blätter 57, 49 (2001)

[35] D. Eliezer, I.I. Kogan, cond-mat/9808240 (1998).

[36] R. Cont, J.-P. Bouchaud, Macroeconom. Dynam. 4, 170 (2000).

[37] J. Bendler, D.G. LeGrand, W.V. Olszewski, in: Transport and Relaxation in Random Materials. Eds. J. Klafter, R.J. Rubin, M.F. Shlasinger, World Sci., Singapore 1986

[38] N.W. Tschoegel, The Phenomenological Theory of Linear Viscoelastic Behavior, Springer-Verlag, Berlin 1989.

[39] Uncertainty and Risk, Eds. M. Abdellaoni, R.D. Luce, M.J. Machina, B. Munier, Springer-Verlag, Berlin 2007.

[40] P. Richmond, S. Hutzler, R. Coelho, P. Reptowicz, in: Econophysics and Sociophysics. Trends and Perspectives, Eds. B.K. Chakrabarti, A. Chakraborti, A. Chatterjee, Wiley-VCH Verlag, Weinheim 2006.

[41] S. Iyazima, K. Yamamoto, in: Proc. Third Nikkei Econophysics Symp., Ed. H. Takayasu Springer-Verlag, Tokyo 2006.

[42] K. Yamamoto, S. Miyazima, H. Yamamoto, T. Ohtsuki, A. Fujihara, in: Proc. Third Nikkei Econophysics Symp., Ed. H. Takayasu, Springer-Verlag, Tokyo 2006.

[43] M. Jagielski, R. Kutner, Acta Phys. Pol. A 117, 615 (2010).

[44] R. Richert, A. Blumen, in: Disorder Effects on Relaxational Processes: Glasses, Polymers, Proteins, Eds. R. Richert, A. Blumen, Springer-Verlag, Berlin 1994.

[45] F. Mainardi, M. Raberto, R. Gorenflo, E. Scales, Physica A 287, 468 (2000).

[46] R. Kutner, F. Świtała, Quantitative Finance 3, 201 (2003). 